\title{
In situ small-angle X-ray and neutron scattering measurements on a blend of deuterated and hydrogenated polyethylenes during uniaxial drawing
}

\begin{abstract}
Go Matsuba ${ }^{1}$, Chie Ito ${ }^{2}$, Yunfeng Zhao ${ }^{1}$, Rintaro Inoue ${ }^{2}$, Koji Nishida ${ }^{2}$ and Toshiji Kanaya ${ }^{2}$
In situ small-angle X-ray and neutron scattering (SAXS and SANS) measurements were conducted on an isotropic blend of deuterated polyethylene and hydrogenated polyethylene (HPE; $3 \mathrm{wt} \%$ ) during uniaxial drawing at $125{ }^{\circ} \mathrm{C}$ to clarify the formation mechanism of the shish-kebab from the isotropic film. In the early stage of drawing, two-spot scattering patterns were observed along the drawing direction in both the SAXS and SANS patterns, and the patterns gradually shifted to lower angles, which indicated that the isotropic lamellar crystals rotate and orient to align and that the long period increases because of the elongation of the amorphous region. In the late stage of drawing, the long-period peak gradually shifted to higher angles and the peak width broadened and finally disappeared at the local drawing ratio $R_{\mathrm{D}} \sim 6.0$, suggesting fragmentation of lamellar crystals (kebabs) and pulling of the polymer chains out of the kebab. However, only the 2D SANS patterns exhibited streak-like scattering in the direction normal to drawing above $R_{\mathrm{D}} \sim 2.4$, suggesting the preferential orientation and stretching of the HPE chains to form the extended chain crystal (shish). By analyzing the SANS data using the multicore-shell cylinder model, the diameter of the shish that consisted of HPE decreased from 9.0 to $4.5 \mathrm{~nm}$ when the drawing was increased from $R_{\mathrm{D}}=3.8$ to 8.0. Polymer Journal (2013) 45, 293-299; doi:10.1038/pj.2012.143; published online 1 August 2012
\end{abstract}

Keywords: polyethylene; shish-kebab; small-angle neutron scattering; small-angle X-ray scattering

\section{INTRODUCTION}

Polymer processing is one of the most important areas of research and development in polymer industries. Consequently, understanding the crystallization process of semi-crystalline polymers under various flows, such as shear, elongation and drawing, and the subsequent orientation and deformation processes of crystals for controlling the higher order structure is of considerable importance. ${ }^{1-5}$ During the crystallization process under flows, the so-called shish-kebab structure, which consists of a long central core (shish) surrounded by lamellar crystals (kebabs), is often observed. It is believed that the shish-kebab is the structural origin of the ultrahigh strength and modulus of fibers; therefore, intensive research studies have been conducted by many research groups with the aim of examining the structure and the formation process of the shish-kebab. ${ }^{6-17}$ Recent research trends include the use of a shear cell with the so-called short-term shear technique. ${ }^{15,18-24}$ This technique is excellent for investigating the formation mechanism of the shish-kebab during the early crystallization stage under mild shear conditions. However, it is known that there are many methods for preparing fibers with the shish-kebab structure that have ultrahigh modulus and strength, such as processing by extruding, ${ }^{3,8-10}$ gel spinning ${ }^{16,17}$ and super drawing of isotropic films. ${ }^{25-28}$ The morphology in polyethylene (PE) prepared by melt extrusion was studied by Keller et al. ${ }^{7-10}$ using transmission electron microscopy (TEM), and the authors observed that the shish was $\sim 10 \mathrm{~nm}$ in diameter and several micrometers in length. Based on the TEM results, it was concluded that the shish is an extended chain crystal and the kebab is a folded chain crystal. A PE gel spun fiber was also studied by Ohta et al. ${ }^{16,17}$ using TEM; the authors reported a similar shish-kebab structure with an ultrahigh modulus and strength fiber. Therefore, many studies have demonstrated that the final morphology in ultrahigh modulus and strength fibers prepared using various methods is the shish-kebab structure although the formation mechanism of the shish-kebab must be different in each type of fiber because the initial structure is considerably different in each method.

In previous works, ${ }^{29}$ we have studied an uniaxially elongated PE blend of deuterated low molecular weight PE and hydrogenated high molecular weight PE (2.8 wt\%) using neutron and X-ray scattering techniques over a wide range of scattering vector, $Q$

\footnotetext{
${ }^{1}$ Department of Polymer Science and Engineering, Faculty of Engineering, Yamagata University, Yonezawa, Japan and ${ }^{2}$ Institute for Chemical Research, Kyoto University, Gokasho, Uji, Japan

Correspondence: Dr G Matsuba, Department of Polymer Science and Engineering, Faculty of Engineering, Yamagata University, 4-3-16 Jonan, Yonezawa 992-8510, Japan. Email: gmatsuba@yz.yamagata-u.ac.jp

or Professor T Kanaya, Institute for Chemical Research, Kyoto University, Gokasho, Uji 611-0011, Japan.

Email: kanaya@scl.kyoto-u.ac.jp
}

Received 5 March 2012; revised 11 June 2012; accepted 12 June 2012; published online 1 August 2012 
$\left(1 \times 10^{-3} \sim 30 \mathrm{~nm}^{-1}\right)$, where $Q=4 \pi \sin \theta / \lambda \quad(2 \theta$ and $\lambda$ are the scattering angle and wavelength, respectively). We previously observed that the fibril structure in the $\mu \mathrm{m}$ scale was formed from a high molecular weight component from the quantitative analysis of the data using the multicore-shell cylinder model, we determined that the fibril structure was $\sim 1 \mu \mathrm{m}$ in radius and approximately about $12 \mu \mathrm{m}$ long and included approximately three extended chain crystals with radius of $\sim 4.5 \mathrm{~nm}$. This study clarified the detailed structure of the shish-kebab in uniaxially elongated PE but unfortunately did not provide direct information about the formation mechanism of the shish-kebab in the elongated fiber. Therefore, in this study, we focus on the structure development in an isotropic PE film during drawing at a temperature just below the nominal melting temperature, $T_{\mathrm{m}}$, to clarify the shish-kebab formation mechanism. For this purpose, we prepared a PE blend of deuterated and hydrogenated PE and performed in situ small-angle X-ray and neutron scattering (SAXS and SANS) measurements during drawing. The SAXS patterns were analyzed to clarify the formation mechanism of the kebab (oriented lamellar crystals), and the 2D SANS patterns were analyzed using the multicore-shell cylinder model to clarify the shish formation mechanism from an isotropic film.

\section{EXPERIMENTAL SECTION}

We used hydrogenated PE (HPE) supplied from Mitsui Chemical Inc., Tokyo, Japan $\left(M_{\mathrm{w}}=2000000\right.$ and $\left.M_{\mathrm{w}} / M_{\mathrm{n}}=12\right)$ and deuterated PE (DPE) bought from Cambridge Isotope Laboratories, Inc., Andover, MA, USA $\left(M_{\mathrm{w}}=600000\right.$ and $\left.M_{\mathrm{w}} / M_{\mathrm{n}}=2\right)$ in this experiment. The nominal melting temperatures of HPE and DPE determined by differential scanning calorimetry measurements were 131 and $126^{\circ} \mathrm{C}$, respectively, at a heating rate of $5^{\circ} \mathrm{C} \mathrm{min}{ }^{-1}$. The blend of HPE and DPE was prepared as follows. The two PEs were dissolved in hot $o$ dichlorobenzene with an antioxidant reagent (2,6-t-butyl-p-cresole) to form a homogeneous solution at $145^{\circ} \mathrm{C}$ under a nitrogen atmosphere. After maintaining the solution at $145^{\circ} \mathrm{C}$ for $2 \mathrm{~h}$, it was poured into methanol to precipitate the blend. The blend sample was filtered from $o$-dichlorobenzene and rinsed with methanol. The precipitate was vacuum-dried at room temperature for several days. The dried samples were hot-pressed at $170{ }^{\circ} \mathrm{C}$ for $3 \mathrm{~min}$ and rapidly quenched in ice water to obtain a film that was $\sim 0.5 \mathrm{~mm}$ thick. The concentration of HPE in the blend was $3 \mathrm{wt} \%$.

In situ SANS measurements were performed using the SANS-U spectrometer at the JRR-3 reactor, ${ }^{30}$ Japan Atomic Energy Agency, Tokai, Japan. In the SANS-U spectrometer, the scattering vector, $Q$, range was $0.07-0.7 \mathrm{~nm}^{-1}$. The neutron wavelength $(\lambda)$ was $0.7 \mathrm{~nm}$, and the dispersion $(\Delta \lambda / \lambda)$ was $10 \%$. The scattered neutrons were detected using a two-dimensional position sensitive detector that had an area of $65 \times 65 \mathrm{~cm}^{2}\left(128 \times 128\right.$ pixel $\left.^{2}\right)$. In situ SAXS measurements were performed on the BL-15A beamline $e^{31}$ at the Photon Factory, KEK, Tsukuba, Japan. The sample-to-camera distance was $2.1 \mathrm{~m}$, and the X-ray wavelength was $0.15 \mathrm{~nm}$. A CCD camera (C7330: Hamamatsu Photonics K.K., Hamamatsu, Japan) with an image intensifier was used as the detector for the in situ SAXS measurements. The $Q$ range was $0.08-1.5 \mathrm{~nm}^{-1}$. The exposure times were 300 and $2 \mathrm{~s}$ for the SANS and SAXS measurements, respectively. The SANS and SAXS patterns were recorded every $5 \mathrm{~min}$ and $10 \mathrm{~s}$, respectively. The two-dimensional (2D) SANS and SAXS patterns were evaluated after correcting the raw data for the sample thickness and background. For the analysis of the SANS and SAXS patterns, we calculated the sector-averaged intensities parallel and normal to the drawing direction from the $2 \mathrm{D}$ profiles in azimuthal angle ranges between $-20^{\circ}$ and $+20^{\circ}$ and between $+70^{\circ}$ and $+110^{\circ}$ to evaluate the oriented structure.

The films were drawn at $125^{\circ} \mathrm{C}$ using a homemade drawing machine in both the SANS and SAXS spectrometers. Before drawing, the sample was crystallized at $125^{\circ} \mathrm{C}$ for $20 \mathrm{~min}$ in the sample cell, which ensured that each experiment was conducted with samples that had the same thermal history. We selected this temperature $\left(125^{\circ} \mathrm{C}\right)$ because it is above the so-called $\alpha_{c}$ transition temperature $T_{\alpha \mathrm{c}}\left(\sim 75^{\circ} \mathrm{C}\right)$ in $\mathrm{PE},{ }^{32}$ above which $\mathrm{PE}$ chains can translate inside the crystal. Therefore, we expected that the chains were pulled out from the crystals during the drawing process. ${ }^{16}$ The initial sample length was $20 \mathrm{~mm}$. Drawing at $125^{\circ} \mathrm{C}$ was performed at a rate of $6 \mu \mathrm{ms}^{-1}$ in the drawing machine; therefore, the average strain rate was $3.0 \times 10^{-4} \mathrm{~s}^{-1}$. The local drawing ratio, $R_{\mathrm{D}}$, in the irradiated position of the specimen was determined by measuring the displacement of marks on the specimen at intervals of $1 \mathrm{~mm}$ before drawing. In this condition, it took $\sim 140 \mathrm{~min}$ to finish the drawing up to $R_{\mathrm{D}}=8.0$ at the irradiated position of the specimen. Therefore, the effective strain rate was $8.3 \times 10^{-4} \mathrm{~s}^{-1}$. Note that we did not observe necking during the drawing process.

\section{RESULTS AND DISCUSSION}

Figure 1 presents the time evolution of the 2D SANS and SAXS patterns during the drawing process at $125^{\circ} \mathrm{C}$ at various drawing ratios, $R_{\mathrm{D}}$ s. The SANS and SAXS patterns at $R_{\mathrm{D}}=1.0$ before drawing are isotropic and exhibit a broad scattering maximum at approximately $Q=0.12 \mathrm{~nm}^{-1}$. This broad peak is a well-known long-period peak and corresponds to the spacing between the lamellar crystals. The isotropic patterns indicate that the lamellar crystals are isotropically distributed in the sample. Note that the SANS and the SAXS were almost identical before drawing, which indicates that the HPE chains were homogeneously distributed in the DPE. At $R_{\mathrm{D}}=1.8$, two-spot scattering patterns in the direction parallel to drawing were observed in both the SANS and SAXS measurements. This result must be due to the orientation of the lamellar crystals to align along the drawing direction. The oriented lamellar crystals become the so-called kebabs; therefore, the oriented lamellar crystals were called kebabs hereafter. At $R_{\mathrm{D}}=2.4$, not only the two-spot scattering from the kebabs but also streak-like scattering in the normal direction to drawing were observed in the SANS pattern although the intensity of the streak-like scattering was weak. However, only the two-spot scattering from the kebabs was observed in the SAXS pattern. These results can be understood by considering the difference of scattering

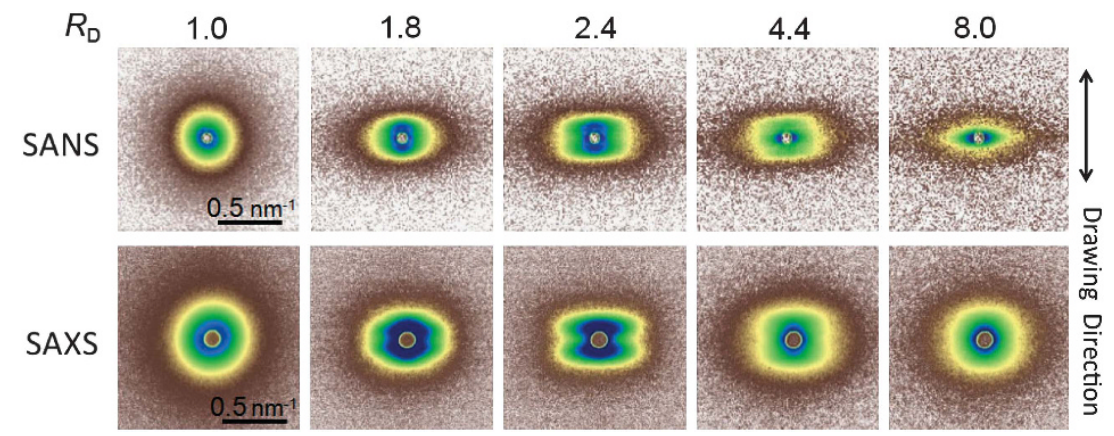

Figure $12 \mathrm{D}$ SANS and SAXS patterns for various drawing ratios, $R_{\mathrm{D}}$. The drawing direction is longitudinal. 
contrasts between neutrons and X-rays. It is well known that the neutron scattering contrast is determined by the scattering length density difference, ${ }^{33}$ but the X-ray scattering contrast is dominated by the electron density difference. ${ }^{34}$ Therefore, in the DPE and HPE blend, the neutron scattering contrast is determined by the density fluctuations between the crystalline and amorphous phases and the composition fluctuations between DPE and HPE. However, the X-ray scattering contrast is only dominated by the density fluctuations between the crystalline and amorphous phases. Therefore, it is believed that the streak-like scattering in the SANS patterns was caused by the preferential orientation of HPE parallel to the drawing direction. This preferential orientation must be due to the higher molecular weight of HPE $\left(M_{\mathrm{w}}=2000000\right)$ compared with DPE $\left(M_{\mathrm{W}}=600000\right)$, and the oriented structure must develop into the socalled shish. ${ }^{29}$ At $R_{\mathrm{D}}=4.4$, the two-spot scattering from the kebabs weakened in intensity and broadened in width in the direction normal to the drawing in both the SAXS and SANS patterns, although the streak-like scattering became distinct in the SANS pattern. When the drawing ratio was increased to $R_{\mathrm{D}}=8.0$, the two-spot scattering disappeared in the SANS and SAXS patterns, suggesting that the kebabs disappeared and the shish grew. A similar disappearance of the kebabs has been reported in PE fibers prepared using a gel spinning method during drawing by Ohta et al. ${ }^{17}$ The streak-like scattering normal to the drawing direction, however, became stronger only in the SANS patterns, which indicates that more HPE chains were participating in the shish. We attempted to evaluate the fraction change of HPE during the drawing, but it was impossible to estimate because the scattering intensity depended on both the scattering contrast and the number of the shish (or the total volume fraction of shish); it was extremely difficult to separate the two contributions during the drawing process. In principle, the shish would be observed even in the SAXS measurements, but it was not observed during this experiment. This result must be because the small number of shish crystals were below the threshold for detection. We conducted SANS measurements during the heating process after the drawing and observed that the highest melting temperature that corresponded to the streak-like scattering was $\sim 141^{\circ} \mathrm{C}$, which was close to the equilibrium melting temperature. This observation confirms that the streak-like scattering includes extended chain crystals (or shishs), although there were not many crystals.

For a more detailed analysis of the orientation and disappearance of the lamellar crystals (or the kebabs), we calculated the 1D SAXS profiles along the direction parallel to drawing because we saw the density fluctuations between the lamellar crystal and amorphous phases in the SAXS but not the composition fluctuation between DPE and HPE. The calculated 1D SAXS profiles are plotted in Figure 2 for various drawing ratios after correcting for the Lorentz-factor $\left[I(Q) \cdot Q^{2}\right] .^{35}$ The long period, $L$, was evaluated from the peak positions, $Q_{\text {peak }}$, through $L=2 \pi / Q_{\text {peak }}$ and plotted in Figure 3 with the peak intensity, $I_{\text {peak }}$, as a function of the drawing ratio. The long period, $L$, and the peak intensity, $I_{\text {peak }}$, increased with drawing in the low $R_{\mathrm{D}}$ region, and after presenting a maximum at $R_{\mathrm{D}} \sim 1.8$, both of the parameters decreased with drawing and the peak finally disappeared at $R_{\mathrm{D}} \sim 6.0$. During the early stage of drawing below $R_{\mathrm{D}} \sim 1.8$, the isotropically distributed lamellar crystals rotated and oriented along the parallel direction due to the drawing, then the isotropic scattering pattern became the anisotropic two-spot pattern. To quantitatively evaluate the orientation of the lamellar crystals, we calculated the ratio of the integrated intensity of the parallel direction to that of the normal one $\left(I_{\text {parallel }} / I_{\text {normal }}\right)$ in the range of $Q=0.08-0.15 \mathrm{~nm}^{-1}$. This ratio is a measure of the orientation. The

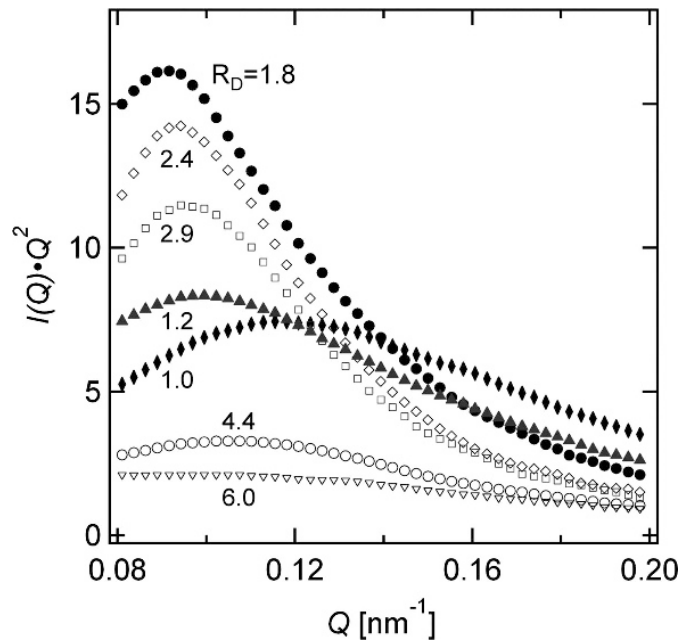

Figure 2 SAXS intensity, $I(Q) \cdot Q^{2}$, in the direction parallel to drawing for various drawing ratios, $R_{\mathrm{D}}$, after correcting for the Lorentz-factor.

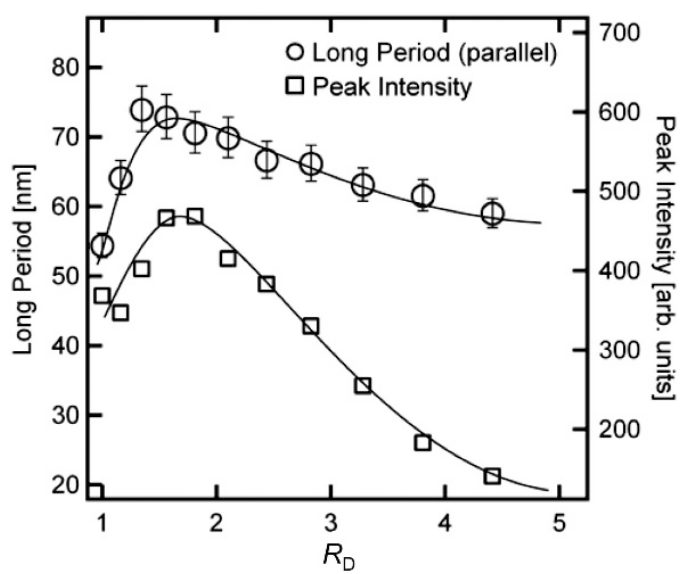

Figure 3 Long period, $L$, and peak intensity, $I_{\text {peak, }}$ as a function of the drawing ratio, $R_{\mathrm{D}}$.

result is shown in Figure 4 as a function of $R_{\mathrm{D}}$. The ratio began to increase with the drawing ratio above $R_{\mathrm{D}} \sim 1.5$, which indicated that the lamellar crystals began to rotate and orient to align to the stretching direction. According to the anisotropic change of the scattering pattern, the peak intensity becomes stronger because many lamellar crystals (or kebabs) orient in the same direction. In this process, the long period, $L$, of parallel direction became longer, likely because of the elongation of the amorphous region between the lamellar crystals because of the drawing. In addition, the width of the scattering peak became narrow in the parallel direction, as observed in Figure 2, which suggests that the distribution of the long period, $L$, in the parallel direction became narrow. On further drawing above $R_{\mathrm{D}} \sim 1.8$, the long period, $L$, and the peak intensity, $I_{\text {peak }}$, decreased with drawing. This result could be explained in terms of lamellar fragmentation. $^{36-38}$ When tie chains in the amorphous region between the lamellar crystals are fully stretched during the drawing process, a large stress is exerted on the lamellar crystals. In this situation, the lamellar crystals must be broken into two or more pieces (fragmentation), resulting in a reduction in the long period, $L$. The distribution of the long period, $L$, must be broader, thereby resulting in a broader width of the scattering peak in the parallel 


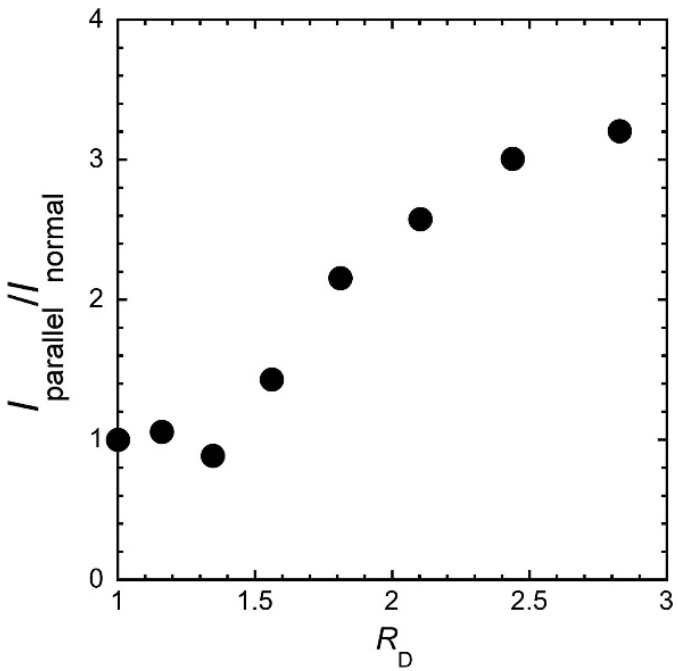

Figure 4 Ratio of the integrated SAXS intensity for the parallel direction to that for the normal one in the range of $Q=0.08-0.15 \mathrm{~nm}^{-1}$.

direction above $R_{\mathrm{D}} \sim 3.0$, as observed in Figure 2. Furthermore, the reduction in the lateral size of the fragmented lamellar crystals caused the broadening of the peak width in the normal direction, which is observed in Figure 1. Both of the broadenings weakened the peak intensity. These facts agree with the experimental observations. In the high drawing ratio region above $R_{\mathrm{D}} \sim 6.0$, the long-period peak disappears, as observed in Figure 2. In other words, the kebabs might disappear with drawing. In this region, the lamellar crystals were further fragmented into smaller crystallites. Finally, polymer chains in the lamellar crystals could be pulled out because the temperature $\left(125^{\circ} \mathrm{C}\right)$ was above the $\alpha_{\mathrm{c}}$ transition temperature $T_{\alpha \mathrm{cc}}{ }^{32}$ Hiss et al..$^{36}$ insisted that polymer chains were disentangled in high drawing ratios. It is therefore expected that the pulled and disentangled chains are incorporated into the extended chain crystal (or the shish) in the $\mathrm{nm}$ length scale. ${ }^{39,40}$ To confirm this expectation, we analyzed the SANS data with a focus on the streak scattering normal to the drawing direction.

As previously mentioned, the streak scattering was observed in the direction normal to the drawing only in the SANS pattern (see Figure 1) in the drawing range above $R_{\mathrm{D}}=2.4$. This result suggests that the shish is primarily formed by the high molecular weight HPE; therefore, analyzing the SANS data in the normal direction will yield information about the formation of the shish structure during the drawing process.

In our previous study, ${ }^{29}$ we performed SANS and SAXS measurements on a similar blend of HPE and DPE over a wide $Q$ range $\left(1 \times 10^{-3} \sim 30 \mathrm{~nm}^{-1}\right)$ using various scattering instruments, and we observed that the streak scattering originated from fibril structures in the $\mu \mathrm{m}$ scale, including some extended chain crystals (the shishs) in the nm-scale ${ }^{29}$ based on the analyses using the multicore-shell cylinder model. The origin of the fibril structure in the $\mu \mathrm{m}$ scale is not currently clear, but one possibility is that the entangled HPE network is stretched and split into bundles in the direction normal to drawing. We have checked the phase separation between HPE and DPE at $190{ }^{\circ} \mathrm{C}$ for $2 \mathrm{~h}$, but could not find any signs of the phase separation. In the present work, however, the $Q$ range is limited due to the in situ measurements. Therefore, we cannot directly evaluate the fibril structure on the $\mu \mathrm{m}$ scale, but we assume that the streak-like scattering originated from the fibril structure, including the shish structure in the $\mathrm{nm}$ scale consisting of HPE, similar to the previous work. ${ }^{29}$ It was further assumed that the extended chain crystals and fibrils consisted of HPE and a mixture of HPE and DPE, respectively, and that the fibrils were surrounded by a matrix of DPE. Under these assumptions, the scattering intensity of the model could be approximated by

$$
I_{\mathrm{SANS}}(Q)=\left(\rho_{\mathrm{cD}}-\rho_{\mathrm{aD}}\right)^{2} I_{\mathrm{den}}(Q)+\left(\rho_{\mathrm{cH}}-\rho_{\mathrm{aD}}\right)^{2} I_{\mathrm{row}}(Q)+I_{\text {inc }}
$$

where $\rho_{\mathrm{cD}}, \rho_{\mathrm{aD}}$ and $\rho_{\mathrm{cH}}$ are the scattering length densities of DPE in the crystalline region, DPE in the amorphous region and HPE in the crystalline region, respectively, and $I_{\mathrm{den}}(Q), I_{\text {row }}(Q)$ and $I_{\text {inc }}$ are the structure factor due to the density fluctuations, the form factor of the fibril including the extended chain crystals (shishs) in the nm scale, and the incoherent scattering intensity, respectively. The first term corresponds to the Fourier transform of the density correlation functions, therefore $I_{\mathrm{den}}(Q)$ can be observed in the SAXS measurement, and equation (1) is written as

$$
I_{\mathrm{SANS}}(Q)=K I_{\mathrm{SAXS}}(Q)+\left(\rho_{\mathrm{cH}}-\rho_{\mathrm{aD}}\right)^{2} I_{\text {row }}(Q)+I_{\text {inc }}
$$

where $K$ is a ratio of contrast factor of neutron scattering to $\mathrm{X}$-ray scattering. In this procedure, we neglected the incoherent scattering contribution mainly from hydrogen atoms in HPE because the concentration of HPE was very small, as shown previously. ${ }^{29}$

We evaluated the contrast factor, $K$, by comparing the neutron and $\mathrm{X}$-ray scattering intensities in the high $Q$ range above $10 \mathrm{~nm}^{-1}$ in our previous paper. ${ }^{29}$ The X-ray intensity was adjusted to the neutron intensity such that the intensity ratio of the wide angle $\mathrm{X}$-ray scattering to the wide angle neutron scattering was equal to the calculated ratio. In this in situ experiment, we only performed the $\mathrm{X}$-ray and neutron-scattering measurements over a limited $Q$ range, and we did not have neutron and X-ray scattering data in a high $Q$ region above $10 \mathrm{~nm}^{-1}$. Therefore, we used the same contrast factor value for the neutron scattering to X-ray scattering as before. ${ }^{29}$ Using the contrast factor, we calculated the $\mathrm{X}$-ray scattering intensity relative to the neutron intensity in both the parallel and normal directions. After subtracting the X-ray data, the SANS intensities were plotted at $R_{D}=6.0$ and 8.0 in Figures $5 \mathrm{a}$ and $\mathrm{b}$, respectively. The SANS intensities corresponds to the second term in equation (2). Note that the SAXS intensity is lower than the SANS intensity by more than one order of magnitude in the normal direction, and hence, the effect of the subtraction is not significant. However, the SAXS intensity is almost comparable with the SANS intensity in the parallel direction, especially in the high $Q$ range above $0.2 \mathrm{~nm}^{-1}$, and the subtraction was not successful; therefore, we excluded the data above $Q=0.2$ $\mathrm{nm}^{-1}$. The strong streak scattering in the normal direction in the SANS patterns must be mainly due to the shish structure composed by HPE, and could be regarded as a form factor of the fibril in the $\mu \mathrm{m}$ scale including the nm-scale shish (second term in equation (2)). To describe the form factor, we employed the multicore-shell cylinder model. ${ }^{29}$ Although the multicore-shell cylinder model was explained in the previous paper, ${ }^{29}$ we recall here the essence of the model. The schematic drawing of the model is shown in Figure 6. In the model, the fibril structure that consists of a mixture of HPE and DPE is expressed by a large cylinder with a diameter of $2 R_{\text {shell }}$ and a height of $2 H$. The fibril includes some extended chain crystals (or shishs) of $\mathrm{HPE}$, which are expressed by core cylinders with a radius of $2 R_{\text {core }}$ and a height of $2 H$. The number of the core cylinders is given by $n$. Neglecting the cross terms between the cores and between the core and shell in this model, the scattering intensity could be 

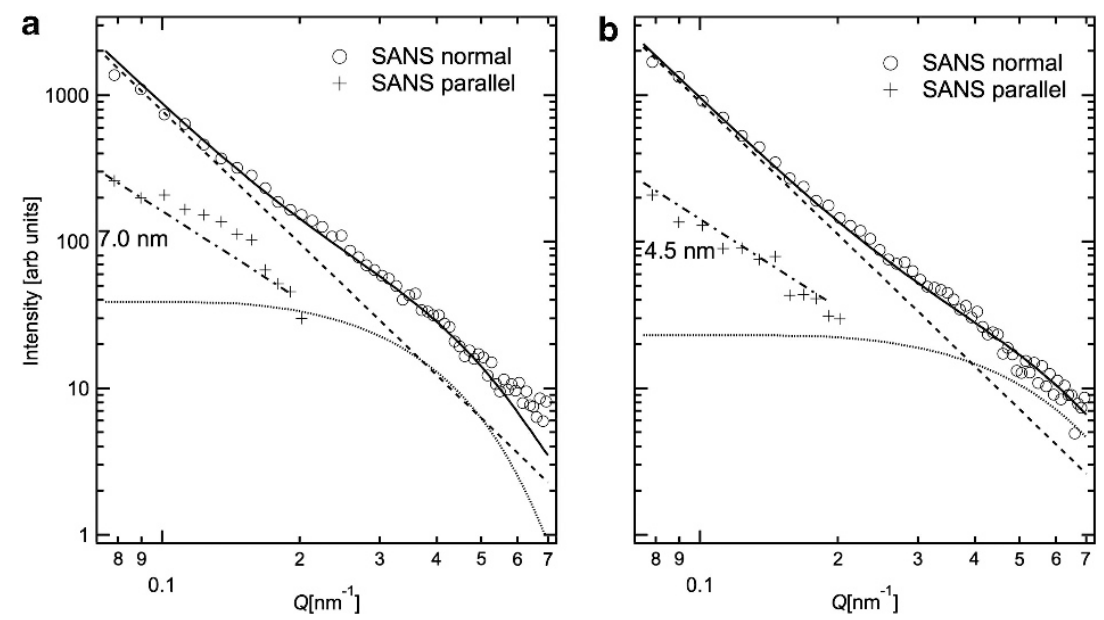

Figure 5 1D SANS intensities after subtracting the density fluctuation contributions in the parallel and normal direction to drawing for a blend of DPE and HPE (3 wt \%) at $125^{\circ} \mathrm{C}$. The drawing ratios are $R_{\mathrm{D}}=6.0$ (a) and 8.0 (b). The solid line is the result of fit with the multicore-shell cylinder model to the SANS intensity in the normal direction, and dashed and dotted lines are the contributions from the shell cylinder with $R_{\text {shell }}=1 \mu \mathrm{m}$ and the core cylinder with $R_{\text {core }}=4.5 \mathrm{~nm}$, respectively. Chain line is the calculated intensity in the parallel direction from multicore-shell cylinder model with $2 \mathrm{H}=12 \mu \mathrm{m}$ and $R_{\text {shell }}=1 \mu \mathrm{m}$.

approximated by

$$
\begin{aligned}
I_{\text {core-shell }}(Q)= & {\left[V_{\text {shell }}\left(\rho_{\text {shell }}-\bar{\rho}_{D}\right) F\left(Q, R_{\text {shell }}, H\right)\right]^{2} } \\
& +\left[n V_{\text {core }}\left(\rho_{\text {core }}-\rho_{\text {shell }}\right) F\left(Q, R_{\text {core }}, H\right)\right]^{2}
\end{aligned}
$$

where $F(Q, R, H)$ is scattering amplitude of a cylinder with a length of $2 H$ and a radius of $R$, reported by Shibayama et al. ${ }^{41} V_{\text {shell }}$, $V_{\text {core }}, R_{\text {shell }}$ and $R_{\text {core }}$ are the shell and core volumes and the shell and core radii, respectively, and $\rho_{\text {shell, }}, \rho_{\text {core }}$ and $\bar{\rho}_{D}$ are the scattering length densities in the shell, the core, and the DPE matrix, respectively. Following the previous procedure, ${ }^{29}$ we assumed $\rho_{\text {core }}=\rho_{\mathrm{cH}}$ and $\rho_{\text {shell }}$ was calculated assuming that the shell volume fraction was 0.3 and that the HPE was homogeneously distributed in the shell with the degree of crystallinity of 0.63 , which were determined by the differential scanning calorimetry data. $\bar{\rho}_{D}$ was calculated under the assumption that the matrix only consisted of DPE. Note that the shell volume fraction of 0.3 was determined in the fitting procedure as follows. ${ }^{29}$ It was assumed that the HPEs were distributed homogeneously in the shell. Under these assumptions, we calculated $\rho_{\text {shell }}$ after we determined the volume fraction of the shell. In the multicore-shell cylinder model, the intensity is given by equation (3); therefore, the intensity ratio at $Q=0$ is $R=\left[V_{\text {shell }}\left(\rho_{\text {shell }}-\bar{\rho}_{D}\right)\right]^{2} /\left[n V_{\text {core }}\left(\rho_{\text {core }}-\rho_{\text {shell }}\right)\right]^{2}$. Extrapolating to $Q=0$, we determined the ratio $R$ of $\sim 10^{-7}$, corresponding to the shell volume fraction of 0.3 . As mentioned previously, the $Q$ range in this experiment was limited; therefore, it was impossible to determine the radius, $R_{\text {shell }}$ and height, $2 H$, in the $\mu \mathrm{m}$ scale directly. As we could not determine these values directly, we used the same values of $R_{\text {shell }}=1 \mu \mathrm{m}$ and $2 H=12 \mu \mathrm{m}$ as those in the previous report. ${ }^{29}$ Similar fibril structures in the $\mu \mathrm{m}$ scale have often been observed with $\mathrm{TEM}^{28,42}$ and scanning electron microscope. ${ }^{43}$ We also observed the fibril structure in the PE blend with a radius of $\sim 1 \mu \mathrm{m}$ in confocal microscope measurements, although the data are not shown here. Note that the value of the $\mu \mathrm{m}$-scale radius $R_{\text {shell }}$ does not affect the size evaluation in the nm-scale shish because the length scales are considerably different.

We first calculated the form factor of a shell cylinder with the average value of $R_{\text {shell }}=1 \mu \mathrm{m}$ and the dispersion of $20 \%$, which are shown by dashed lines in Figures $5 \mathrm{a}$ and $\mathrm{b}$, adjusting the lines to the data below $0.15 \mathrm{~nm}^{-1}$. The line is simply a power law with an exponent of -3 , which corresponds to an asymptotic form factor of an oriented cylinder in the normal direction to the long axis. It is clear that the SANS profiles show excess scattering intensities over the power law suggesting some internal structure that must be the nm-scale shish (the extended chain crystal) with a radius of several nm, which Keller et al. ${ }^{3,7-10}$ reported several decades ago. Then, we fitted the multicore-shell cylinder model to the observed SANS intensity in the normal direction, and the results of fits are shown by solid thick curves in Figures $5 \mathrm{a}$ and $\mathrm{b}$. The results of fits are very good. The nm-scale shish (core cylinder) contribution is shown by dotted lines in Figure 5. From the fit in Figures $5 \mathrm{a}$ and $\mathrm{b}$, we estimated the radii of the core cylinders, which were 7.0 and $4.5 \mathrm{~nm}$ for $R_{\mathrm{D}}=6.0$ and 8.0 , respectively. The numbers of the core cylinders were $\sim 1.5$ in both cases. These results suggest that the $\mu \mathrm{m}$-scale fibril structure with a radius of $\sim 1 \mu \mathrm{m}$ includes $\sim 1.5$ extended chain crystals. Because of this small number, we did not observe the extended chain crystals with the SAXS measurement. Note that the ratio of scattering intensity in the parallel direction to the normal one is a function of the cylinder length, $2 H$, at a given radius $R$. Therefore, we calculated the parallel intensity as a function of $2 H$ for $R_{\text {shell }}=1$ $\mu \mathrm{m}$ (see chain lines in Figure 5), and determined that the intensity calculated for $2 H \sim 12 \mu \mathrm{m}$ is the best to describe the observed intensity. This value is very similar with the previously reported value. $^{29}$

We fitted the calculated scattering function from the multicoreshell cylinder model to the data at different drawing ratios, and evaluated the number, $n$, and the radius, $R_{\text {core }}$ of the core cylinder (nm-scale shish). The results are shown in Figure 7. The radius of the core cylinder decreased with the increased the drawing ratio. This result means that the core cylinder composed of HPE chains became thinner with drawing. However, the number of core cylinders in the fibril structure was almost independent of the drawing ratio. During the drawing process, the core cylinder does not split off to create new core cylinders, rather, it simply thins.

As shown above, the kebab structure gradually disappeared when $R_{\mathrm{D}}>1.8$ and may have merged into the nm-scale shish structure. If this explanation is correct, the shish should increase in diameter. 


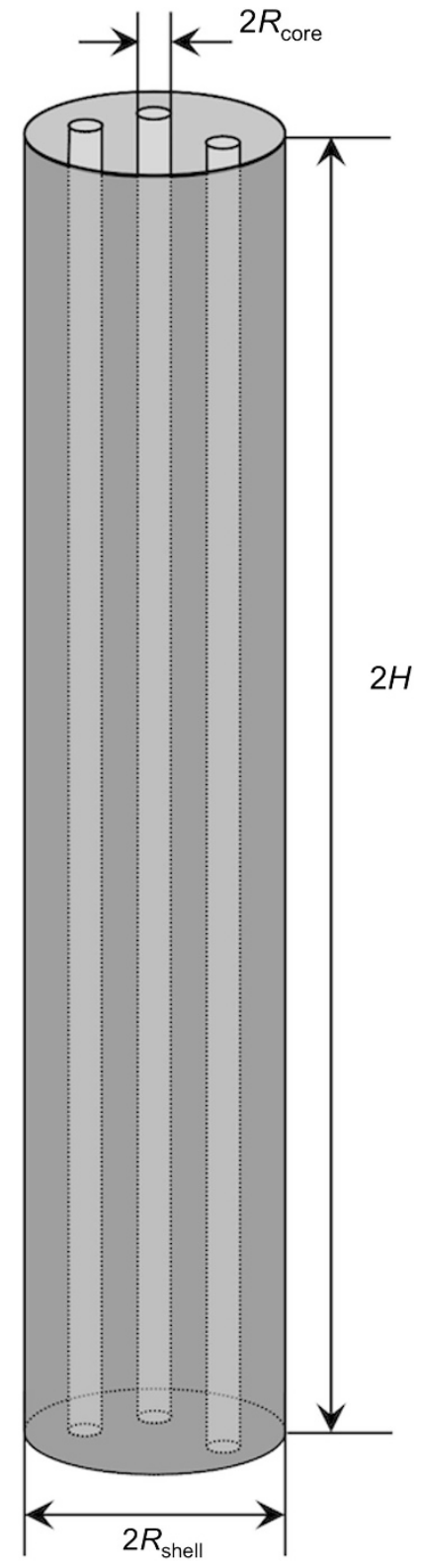

Figure 6 Schematic drawing of the multicore-shell cylinder model.

However, we observe a thinning of the radius of the nm-scale shish structure rather than a thickening. In the early stage of the drawing process, we observed the preferential orientation and extension of high molecular weight HPE to form the shish, suggesting that the shish in the early stage was primarily formed from HPE. On further drawing, the lamellar crystals (the kebabs), which mainly consisted of DPE because of a very high fraction of DPE (97 wt\%), were fragmented into small lamellar crystals. Finally, DPE chains were pulled from the lamellar crystals (the kebabs) to merge onto the shish. In the SANS measurements, we observed the nm-scale shish produced from HPE in the DPE matrix because of the high SANS contrast between HPE and DPE. In the late stage of the drawing process, the DPE chains were merged onto the HPE shish, and they must have been located outside of the HPE shish. The merged DPE chains outside of the HPE shish are almost invisible in the SANS profile. This result may explain why we did not observe a thickening in the shish.

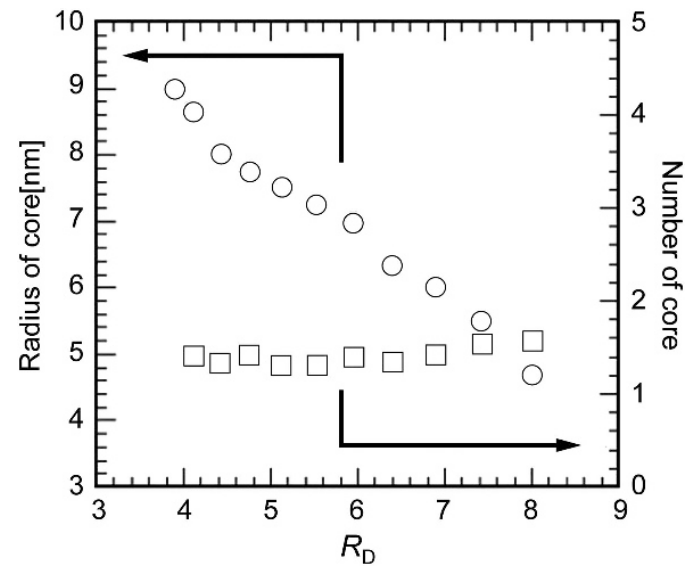

Figure 7 Radius (circle) and number (square) of core cylinders as a function of drawing ratio $R_{\mathrm{D}}$ at $125^{\circ} \mathrm{C}$.

During the drawing process, the visible HPE shish is gradually stretched and thinned, as observed in the SANS measurement.

\section{CONCLUDING REMARKS}

We have studied the structure development of a blend of DPE and HPE (3 wt \%) during the drawing process at a temperature just below the nominal melting temperature using in situ SAXS and SANS measurements. We discussed the development of the isotropically distributed lamellar crystals based primarily on the SAXS data. During the early stage of the drawing process below $R_{\mathrm{D}} \sim 1.8$, the isotropically distributed lamellar crystals rotate and orient to align along the drawing direction. During this process, the amorphous regions between the lamellar crystals are elongated, thereby resulting in the increase of the long period, $L$, in the drawing direction. With further drawing, the lamellar crystals (kebabs) are fragmented into small pieces and the polymer chains are pulled from the kebab, and finally disappeared at $R_{\mathrm{D}} \sim 6.0$. The streak-like scattering appeared in the direction normal to drawing above $R_{\mathrm{D}} \sim 2.4$ only in the in situ SANS measurement, which indicates that the preferential orientation and stretching of HPE chains formed the extended chain crystals (or the shish). The stretching of the HPE chains must be due to the higher molecular weight of HPE compared with DPE, although the average molecular weight of HPE is only three times larger than that of DPE. Combining the SAXS and SANS results, it is concluded that in the late stage of drawing, the kebabs were broken and the polymer chains were pulled from the kebabs and finally merged onto the shish. By analyzing the 1D SANS profiles using multicore-shell model, we observed that the radius of the nm-scale shish that consisted of HPE was approximately $9.0 \mathrm{~nm}$ at $R_{\mathrm{D}}=3.8$, and it decreased to $4.5 \mathrm{~nm}$ at $R_{\mathrm{D}}=8.0$ with further drawing while the number of the shishs was almost constant during the drawing. This result means that the HPE shishs do not separate to form new shishs and that the DPE chains are merged on the outside of the HPE shish in the late stage of drawing, but the DPE on the HPE shish is invisible because of the absence of SANS contrast.

\section{ACKNOWLEDGEMENTS}

This work was conducted under the Joint-use Research Program for Neutron Scattering, Institute for Solid State Physics (ISSP), the University of Tokyo, at the Research Reactor JRR-3, JAEA (Proposal No.7613). 
1 Ward, I. M. Structure and Properties of Oriented Polymers (Wiley, New York, 1975).

2 Ziabicki, A. Fundamentals of Fiber Formation (Wiley, New York, 1976).

3 Keller, A. \& Odell, J. A. The extensibility of macromolecules in solution; A new focus for macromolecular science. Colloid Polym. Sci. 263, 181-201 (1985).

4 Keller, A. \& Kolnaar, J. W. H. Flow-Induced Orientaion and Structure Formation. in Processing of Polymers. (ed. Meijer, H. E. H.) vol. 18, 189-268 (VCH, New York, 1997).

5 Walczak, Z. K. Processses of Fiber Formation (Elsevier, Amsterdam, 2002).

6 Pennings, A. J. Bundle-like nucleation and longitudinal growth of fibrillar polymer crystals from flowing solutions. J. Polym. Sci. Part C Polym. Symp. 59, 55-86 (1977).

7 Odell, J. A., Grubb, D. T. \& Keller, A. A new route to high modulus polyethylene by lamellar structures nucleated onto fibrous substrates with general implications for crystallization behaviour. Polymer 19, 617-626 (1978).

8 Bashir, Z., Odell, J. A. \& Keller, A. High modulus filaments of polyethylene with lamellar structure by melt processing; the role of high molecular weight component. J. Mater. Sci. 19, 3713-3725 (1984)

9 Bashir, Z., Hill, M. J. \& Keller, A. Comparative study of etching techniques for electron microscopy using melt processed polyethylene. J. Mater. Sci. Lett. 5, 876-878 (1986).

10 Bashir, Z., Odell, J. A. \& Keller, A. Stiff and Strong polyethylene with shish-kebab morphology by continuous melt extrusion. J. Mater. Sci. 21, 3993-4002 (1986).

11 Meerveld, J. V., Peters, G. W. M. \& Hutter, M. Towards a rheological classification of flow induced crystallization experiments of polymer melts. Rheol. Acta 44, 119-134 (2004).

12 Baert, J., Puyvelde, P. V. \& Langouche, F. Flow-induced crystallization of PB-1: from the low shear rate region up to processing rates. Macromolecules 39, 9215-9222 (2006).

13 Kimata, S., Sakurai, T., Nozue, Y., Kasahara, T., Yamaguchi, N., Karino, T., Shibayama, M. \& Kornfield, J. A. Molecular Basis of the shish-kebab morphology in polymer crystallization. Science 316, 1014-1017 (2007).

14 Balzano, L., Kukalyekar, N., Rastogi, S., Peters, G. W. M. \& Chadwick, J. C. Crystallization and discussion of flow-induced precursors. Phys. Rev. Lett. 100, 048302 (2008).

15 Balzano, L., Rastogi, S. \& Peters, W. M. Crystallization and precursors during fast short-term shear. Macromolecules 42, 2088-2092 (2009).

16 Hashimoto, T., Murase, H. \& Ohta, Y. A new scenario of flow-induced shish-kebab formation in entangled polymer solution. Macromolecules 43, 6542-6548 (2010).

17 Ohta, Y., Murase, H. \& Hashimoto, T. Structural development of ultra-high strength polyethylene fibers: transformation from kebab to shishs through hot-drawing process of gel-spun fibers. J. Polym. Sci. Part B Polym. Phys. 48, 1861-1872 (2010).

18 Somani, R. H., Hsiao, B. S., Nogales, A., Srinivas, S., Tsuo, A. H., Sics, I., BaltaCalleja, J. \& Ezquerra, T. A. Structure development durng shear flow-induced crystallization of ipp: in-situ small-angle x-ray scattering. Macromolecules 33, 9385-9394 (2000).

19 Fukushima, H., Ogino, Y., Matsuba, G., Nishida, K. \& Kanaya, T. Crystallization of polyethylene under shear flow as studied by time resolved depolarized light scattering. effects of shear rate and shear strain. Polymer 46, 1878-1885 (2005).

20 Ogino, Y., Fukushima, H., Takahashi, N., Matsuba, G., Nishida, K. \& Kanaya, T. Crystallization of isotactic polypropylene under shear flow observed in a wide spatial scale. Macromolecules 39, 7617-7642 (2006).

21 Heeley, E. L., Fernyhough, C. M., Graham, R. S., Olmsted, P. D., Inkson, N. J., Embery, J., Groves, D. J., McLeish, T. C. B., Morgovan, A. C., Meneau, F., Bras, W. \& Ryan, A. J. Shear-induced crystallization in blends of model linear and long-chain branched hydrogenated polybutadienes. Macromolecules 39, 5058-5071 (2006).

22 Keum, J. K., Burger, C., Zuo, F. \& Hsiao, B. S. Probing nucleation and growth behavior of twisted kebabs from shish scaffold in sheared polyethylene melts by in situ X-ray studies. Polymer 48, 4511-4519 (2007).
23 Zhang, C. G., Hu, H. Q., Wang, X. H., Yao, Y. H., Dong, X., Wang, D. J., Wang, Z. G. \& Han, C. C. Formation of cylindrite structures in shear-induced crystallization of isotactic polypropylene at low shear rate. Polymer 48, 1105-1115 (2007).

24 Mykhaylyk, O. O., Chambon, P., Impradice, C., Fairclough, J. P. A., Terrill, N. J. \& Ryan, A. J. Control of structural morphology in shear-induced crystallization of polymers. Macromolecules 43, 2389-2405 (2010).

25 Matsuo, M. \& Sawatari, C. Elastic-modulus of polyethylene in the crystal chain direction as measured by X-ray-diffraction. Macromolecules 19, 2036-2040 (1986).

26 Sawatari, C., Shimogiri, S. \& Matsuo, M. Morphological properties of ultrahigh molecular-weight polyethylene polypropylene blend films produced by gelation crystallization from solutions. Macromolecules 20, 1033-1041 (1987).

27 Sawai, D., Watanabe, D., Morooka, N., Kuroki, H. \& Kanamoto, T. Superdrawing of polytetrafluoroethylene nascent powder by solid-state coextrusion. J. Polym. Sci. Part B Polym. Phys. 44, 3369-3377 (2006).

28 Nakae, M., Uehara, H., Kanamoto, T., Zachariades, A. E. \& Porter, R. S. Structure development upon melt drawing of ultrahigh molecular weight polyethylene: effect of prior thermal history. Macromolecules 33, 2632-2641 (2000).

29 Kanaya, T., Matsuba, G., Ogino, Y., Nishida, K., Shimizu, H. M., Shinohara, T., Oku, T., Suzuki, J. \& Otomo, T. Hierarchic structure of shish-kebab by neutron scattering in a wide q range. Macromolecules 40, 3650-3654 (2007).

30 Ito, Y., Imai, M. \& Takahashi, S. Small-angle neutron scattering instrument of the institute for solid state physics, University of Tokyo (SANS-U). Physica B 213/214, 889-891 (1995).

31 Amemiya, Y., Wakabayashi, K., Hamanaka, T., Wakabayashi, T. \& Hashizume., H. Design of small-angle X-ray diffractometer using synchrotron radiation at the photon factory. Nucl. Instr. Meth. 208, 471-477 (1983).

32 Danch, A., Osoba, W., Chrobak, D., Nowaczyk, G. \& Jurga, S. $\alpha_{c}$ Relaxation of the constrained amorphous phase. J. Therm. Anal. Cal. 90, 201-208 (2007).

33 Bacon, G. E. Neutron Diffraction (Clarendon Press, Oxford, 1975).

34 Guinier, A. \& Fournet, G. Small-angle Scattering of X-rays (Wiley, New York, 1955).

35 Shibayama, M. \& Hashimoto, T. Small-angle X-ray scattering analyses of lamellar microdomains based on a model of one-dimensional paracrystal with uniaxial orientation. Macromolecules 19, 740-749 (1986).

36 Hiss, R., Hobeika, S., Lynn, C. \& Strobl, G. Network stretching, slip processes, and fragmentation of crystallites during uniaxial drawing of polyethylene and related copolymers. A comparative Study. Macromolecules 32, 4390-4403 (1999).

37 Nozue, Y., Shinohara, Y., Ogawa, Y., Takamizawa, T., Sakurai, T., Kasahara, T., Yamaguchi, N., Yagi, N. \& Amemiya, Y. Deformation behavior of banded spherulite during drawing investigated by simultaneous microbeam SAXS-WAXS and POM measurement. Polymer 51, 222-231 (2010).

38 Miyazaki, T., Hoshiko, A., Akasaka, M., Shintani, T. \& Sakurai, S. SAXS studies on structural changes in a poly(vinyl alcohol) film during uniaxial stretching in water. Macromolecules 39, 2921-2929 (2006).

39 Bowden, P. B. \& Young, R. J. Deformation mechanisms in crystalline polymers. J. Mater. Sci. 9, 2034-2051 (1974).

40 Butler, M. F., Donald, A. M. \& Ryan, A. J. Time resolved simultaneous small- and wideangle X-ray scattering during polyethylene deformation 3. Polymer 39, 781-792 (1998).

41 Shibayama, H., Nomura, S., Hashimoto, T. \& Thomas, E. L. Asymptotic behavior and lorentz factor for small-angle elastic scattering profiles from preferentially oriented asymmetric bodies. J. Appl. Phys. 66, 4188-4197 (1989).

42 Kakiage, M. Tamura, T. Murakami, S., Takahashi, H., Yamanobe, T. \& Uehara, $H$. Hierarchical constraint distribution of ultra-high molecular weight polyethylene fibers with different preparation methods. J. Mater. Sci. 45, 2574-2579.

43 Wang, Y., Meng, K., Hong, S., Xie, X. M., Zhang, C. G. \& Han, C. C. Shear-induced crystallization in a blend of isotactic polypropylene and high density polyethylene. Polymer 50, 636-644 (2009). 